\title{
Wave response of enzymatic processes in biological systems of water treatment and treatment of precipitation
}

\author{
Inna Aksenova ${ }^{1, *}$ \\ ${ }^{1}$ Odessa State Academy of Civil Engineering and Architecture, 65000, Marseilles str., 44, Odessa, \\ Ukraine
}

\begin{abstract}
Theoretical studies of the wave response of enzymatic processes in biological systems of wastewater treatment reactors and treatment of precipitation have been carried out. At present, the use of various methods of wave action on the biological systems of water treatment reactors and treatment of precipitation in various types of reactors has been increased to intensify the processes of biological purification and treatment of precipitation. On the basis of this, the hypothesis of wave responses of enzymatic processes arose. The main research issue is to identify the wave response of enzymatic processes in stationary conditions and in the excited state of the system. This observation will allow to determine the limits of wave action on aerobic and anaerobic conversion of organic substrate, hydrolysis of high molecular weight organic compounds, nitrification, denitrification, as well as biological phosphorus removal. The main task of this study is the modeling of multicomponent processes, based on the results of identification and selectivity of the wave response in biological systems of wastewater treatment reactors and treatment of precipitation.
\end{abstract}

\section{Introduction}

Modern reactors for biological water treatment and precipitation treatment are the organized biological engineering systems that transform organic substances into biological mass. The problems of utilization of the biomass obtained, its dehydration, and the environmental safety of the organic product obtained directly depend on the process of its productivity. The priority of efficient operation of biological treatment reactors is the quality of water after facilities; questions about the quality of the biological mass are secondary, and they are resolved in a complex with rare exceptions $[1,2,3,4]$. This is usually related to the variability of the composition of contaminants in the water, the multicomponent and specificity of the colloidal system, causing a variety of engineering solutions. However, a universal engineering solution for identifying microbiological processes in biological treatment reactors and identifying an impact assessment on such a biological system has not been found.

\footnotetext{
*Corresponding author: aksonova.inna@ yandex.ru
} 
Based on the theory of wave oscillations in chemical systems, the paper considers the question of wave responses of the main enzymatic processes that occur in biological water treatment reactors and sediment treatment.

\section{Problem Statement}

The presence of wave excitations in biochemical wastewater treatment processes is determined by the total effect of exo- and endothermic reactions of enzymatic catalytic cleavage of high molecular weight organic compounds. The formation of a colloidal system with specific properties makes it possible to identify the occurrence of wave excitation with respect to the corresponding biochemical process. The identification of processes is not necessary in the practice of modeling microbiological processes in the pharmaceutical and food industries, because the components of the system are known. Therefore, more need to know the kinetics of the process causing the yield and quality of the product. On the contrary, the identification of processes and their stages in biochemical wastewater treatment is very important in order to carry out the process more efficiently. Identification of the quantity and quality of high molecular compounds in waste water is determined by integral methods with chemical consumption of oxygen. The appropriate classification and dynamics of the transformation of organic matter in wastewater are considered in [1].

But this is insufficient to reproduce a more efficient process of reactions of enzymatic catalytic cleavage of high-molecular organic compounds in biochemical reactors of various kinds. Identification of the processes of aerobic and anaerobic conversion of organic substrate, hydrolysis of high molecular organic compounds, nitrification, denitrification, biological removal of phosphorus with wave characteristics makes it possible to recognize processes. The received wave characteristics of waste water for determination of aerobic or anaerobic conversion of organic substrate, hydrolysis of high molecular weight organic compounds, nitrification, denitrification, biological phosphorus removal are compared with model systems in a calm and excited state. The measurement of electrical, thermodynamic characteristics and microscopic examination of the spatial structures of the colloidal microsystem obtained, as well as the dynamics of its changes make it possible to correct the process.

\subsection{Identification of wave phenomena in biological systems of wastewater treatment reactors and treatment of precipitation}

The identification of wave phenomena in reactors of different geometric configurations depends on the hydraulic conditions of the process. Objectively, it should be considered that, in contrast to corridor-type reactors, they differ from wave characteristics in column-type reactors based on the kinetics of the passage of heterogeneous and homogeneous chemical reactions of aerobic or anaerobic conversion of organic substrate, hydrolysis of high molecular organic compounds, nitrification, denitrification, biological phosphorus removal. It should be noted that any biochemical and chemical or physicochemical process has its own wave characteristics. Evaluation of wave characteristics and their comparison with optimal conditions for obtaining a positive result at the outlet from the reactor will make it possible to more efficiently operate the biological systems of reactors for wastewater treatment and treatment of precipitation.

Wave characteristics have also hydrodynamic parameters of fluid motion. Parallel turbodynamic, high-speed, circulating, screw, and combined fluid movements have their own wave characteristics. 
The negative consequences of cavitation, hydraulic shock and their wave characteristics are described in the current literature, but low-frequency oscillations and their wave characteristics are less studied [2].

The positive consequences of low intensity cavitation on biochemical processes give the right to believe that the identification of low-frequency wave phenomena in biological systems of reactors of various kinds will allow modeling of effective conditions for the passage of processes and optimize the management of chemical, biochemical, and physicochemical processes of wastewater treatment and limits of the impact on selfpurification of water bodies.

\subsection{Methods for studying wave responses in biological systems of wastewater treatment and treatment of precipitation}

Methods for studying the state of biochemical processes in the reactor can be based on ultrasonic examination of the corresponding volume of the reactor, thermo-technical examination of the volume of the biological system of the reactor, visualization of the microstructures of the bacterial colonies and their spatial configuration in a calm and excited state, etc. A direct study of the state of operation of the biological system of the reactor gives a more objective assessment of biochemical processes and the possibility of their correction.

The action of electric, magnetic electromagnetic, radioactive, ultrafrequency, infrared radiation to intensify or inhibit the development of biocenosis in the biological system of the reactor on the basis of identification of wave phenomena of biochemical processes allows, perhaps, to dose more precisely according to the changes in the spatial structure of the colloid and microcolloid system in the excited state.

The study of exogenous and endogenous zones in the volume of the biological system of the reactor with the help of thermal inspection is provided with data on the location, propagation, and dynamics of exothermic and endothermic biochemical processes, as well as the intensity of diffusion and temperature equalization processes. It is important to obtain thermodynamic characteristics of the operation of different types of loads, insertions, and other types of differentiation of the volume of the biological system of a reactor for wastewater treatment.

Adaptation of methods for ultrasonic examination of reactor volume for the manifestation of qualitative and quantitative characteristics of wave phenomena of aerobic and anaerobic conversion of organic substrate, hydrolysis of high molecular organic compounds, nitrification, denitrification, biological removal of phosphorus in a calm and excited state of a colloidal and micro colloidal system are also important.

The results of ultrasonic examination of hydrodynamic fluid current and wave impulse characteristics that increase or decrease the exogenous and endogenous zones of the processes of biochemical reactions of aerobic and anaerobic conversion of organic substrate, hydrolysis of high molecular organic compounds, nitrification, denitrification, biological phosphorus removal, which are simultaneously detected by thermo-technical examination of the volume of the biological system of the reactor, are comparable with the integral exponents spruce content of organic matter by the ratio of the parameters of permanganate oxidizability and the chemical process of oxygen, total nitrogen and the chemical process of oxygen, total phosphorus and the chemical process of oxygen, nitrogen of ammonium and nitrite- and nitrate ions. Integral indices of individual relationships and their dependencies, which characterize the corresponding biochemical processes in the biological system of a sewage treatment reactor and wave characteristics of enzymatic-catalytic cleavage reactions of high molecular organic compounds, provide an objective assessment of the reactor operation under given hydrodynamic conditions. The stability of the corresponding zones in different hydrodynamic conditions is determined by the rate of metabolic processes and the 
growth of the number of colonies of bacteria, their stability in the fluid flow, adaptation of microorganisms to the corresponding volume of the reactor in the free and attached state. The greater the differentiation of reactor volume is, the more favorable conditions for the passage of aerobic and anaerobic conversion reactions of organic substrate, hydrolysis of high molecular weight organic compounds, nitrification, denitrification, biological phosphorus removal are, and the more uniform the thermal characteristics of the total volume of wastewater treatment reactors and the treatment of precipitation are.

- Modern methods of diagnostics of various types of radiation make it possible to use them for identification of wave phenomena of the biochemical wastewater treatment processes.

- Identification of wave characteristics of aerobic and anaerobic conversion reactions of organic substrate, hydrolysis of high molecular weight organic compounds, nitrification, denitrification, biological phosphorus removal in comparison with integral identification methods make it possible to obtain techniques for qualitative and quantitative characterization of the environment of wastewater treatment reactors and treatment of precipitation.

- Identification of wave phenomena of the hydrodynamic current of a liquid will allow to react in time to radiation that inhibits biochemical processes in the reactor.

- Identification of the characteristics of wave phenomena of biochemical wastewater treatment processes with the development of appropriate techniques will make it possible to more efficiently manage the processes during operation of reactors for wastewater treatment and treatment of precipitation and also to create positive wave characteristics for various types of low-intensity emissions.

- Identification of wave phenomena in the reactions of aerobic and anaerobic conversion of organic substrates, hydrolysis of high molecular weight organic compounds, nitrification, denitrification, biological phosphorus removal in combination with integrated analytical methods of investigation will make it possible to expand the nomenclatural characteristics of the organic matter of large wastewater and rather adapt the corresponding biocenosis.

- Identification of the characteristics of wave phenomena that inhibit the metabolic processes of the corresponding bacteria to regulate their development in biological reactor systems at different stages of purification is useful. In this aspect, a more detailed study of the wave characteristics of the processes of low-frequency cavitation and the action of various types of aeration systems in sewage treatment reactors may be of interest.

- Identification of wave phenomena of the hydrodynamic interaction of a fluid flow with inserts and various kinds of loads will allow to determine their favorable configuration and spatial arrangement.

Investigations of the wave characteristics of biochemical wastewater treatment processes open the prospects for a complex modulation of effective biological sewage treatment using nanotechnology.

\section{Research Questions}

The main question of the research is the identification of the wave response of enzymatic processes under stationary conditions and in the excited state of the system, which will allow us to determine the limits of the wave action on the reactions (aerobic and/or anaerobic conversion of organic substrate, hydrolysis of high molecular weight organic compounds, nitrification, denitrification, biological phosphorus removal). In addition, this study determines the intensity of wave response in biological systems of wastewater treatment reactors and treatment of precipitation and the probability of maximum kinetic parameters of the identified processes. 


\section{Purpose of the Study}

The purpose of the study is to substantiate the hypothesis of the existence of wave responses of homogeneous processes of enzymatic reactions of aerobic and/or anaerobic conversion of organic substrate, hydrolysis of high molecular weight organic compounds, nitrification, denitrification, biological phosphorus removal in biological systems of wastewater treatment reactors and treatment of precipitation.

Identification of processes in biological systems of wastewater treatment reactors and treatment of sediments determines the limits of the influence of various types of impacts on these systems.

\section{Research Methods}

The use of ultrasonic methods for studying biological systems of reactors to identify the reactions of aerobic and anaerobic conversion of an organic substrate, hydrolysis of high molecular weight organic compounds, nitrification, denitrification, biological phosphorus removal makes it possible to monitor the change in the state of the system; formation of microcolloid structures [6], their destruction during the decomposition of organic matter; the formation of excess biomass, the emergence of microcurrents and swirled flows in water treatment and treatment of precipitation, and other characteristics that are of a wave nature.

\subsection{Resonance phenomena in the ultrasonic investigation of wave responses in biological systems of wastewater treatment reactors and treatment of precipitation}

Wave characteristics of changes in the density of a homogeneous medium that arise during aerobic and anaerobic conversion of an organic substrate, hydrolysis of high molecular weight organic compounds, nitrification, denitrification, biological phosphorus removal during ultrasound investigation can cause resonance phenomena.

At resonance frequencies, the electrical impedance has the form [11]:

$$
Z_{r}=\frac{1}{f \omega C_{0}}(1+\theta)
$$

where $\theta=8 \eta k^{2} Q \sin \left(\pi f / 2 f_{Q}\right) / f\left(k_{1} d\right)^{2}, C_{0}$ - piezoceramic capacity, $Q$ - fractionality of resonator, $\omega=2 \pi f, \mathrm{f}-$ frequency, $f_{Q}=c_{1} / 2 d$;

$\mathrm{kt}$ - coefficient of electromechanical coupling;

$\eta$ - compatible oscillation factor of piezoceramic plates;

$\mathrm{k}_{1}, \mathrm{~d} \mathrm{c}_{1}$ - wave number, thickness, and ultrasonic velocity for the piezoceramic plate, respectively.

Wave characteristics of changes in the density of a homogeneous medium that arise during aerobic and anaerobic conversion of an organic substrate, hydrolysis of high molecular weight organic compounds, nitrification, denitrification, biological phosphorus removal during ultrasound investigation can cause resonance phenomena.

At resonant frequencies, the electrical impedance is

$$
U_{S}=\frac{2 h^{2} D \gamma}{k_{1} c^{2}} \cdot \frac{\sin \left(k_{1} \frac{d}{2}\right)}{A S}
$$

where $U_{s}$ - amplitude of electric voltage arising on the receiving piezoceramic plate in the absence of electrical load, h - piezoelectric constant, D - amplitude of electrical induction in an excited piezoceramic plate, $\gamma=\frac{\rho_{2} \mathrm{c}_{2}}{\rho_{1} \mathrm{c}_{1}}$ - the modulus of elasticity of a piezoceramic plate at a constant, $c_{1}$ i $c_{2}$ - ultrasound velocity in piezoelectric plates and liquid, $\rho_{1}$ i $\rho_{2}$ - density of 
piezoelectric and liquid, A, S - wave number and thickness of the liquid layer. At resonance, expression (5) is

$$
\frac{U}{U_{0}}=\frac{\delta \theta}{1+\theta}
$$

where $U$ is an amplitude of electric voltage being taken from the receiving piezoceramic plate, $U_{0}$ - amplitude of excitation voltage, $\theta$ is defined by formula (4), $\delta=Z_{e л} /\left(Z_{e n}+1 /\left(f \omega C_{0}\right)\right.$ is the transmission coefficient for voltage with the acoustics of the circuit to the electrical, and $Z_{e л}$ is a complex resistance of electric load. From the formula obtained it follows that the output voltage is always less than the exciting voltage.

\subsection{Registration of wave responses in biological systems of wastewater treatment and treatment of precipitation}

The use of equivalent surveillance cameras, which are installed directly into the scope of the facility, is proposed for the registration of wave responses in reactors. Equivalent cameras are equipped with sensors to record changes in volumetric, induction, optical, ultrasonic, thermal, and other parameters.

Currently, the market of remote devices for recording technological processes in industry provides unlimited possibilities for using such devices to control enzymatic processes in biological wastewater treatment reactors and to treat precipitation. Sensitivity of sensors and software is oriented to an equivalent volume of stationary conditions for the passage of enzymatic processes; the volume, induction, optical, ultrasonic, thermal data of the wave responses of processes are recorded in accordance with model parameters.

Modern bioreactors for sewage treatment and treatment of precipitation have separate geometric spaces for each stage: aerobic conversion of organic substances, nitrification, denitrification, anaerobic conversion of organic substances; biological isolation of phosphorus. Therefore, the registration of wave reviews of enzymatic processes using equivalent cameras with a corresponding set of sensors is simplified in comparison with the structures in which these zones need to be determined. To determine the zones of the corresponding process in the biological treatment facilities of the classical type, the use of the equivalent chamber method is also possible.

\section{Findings}

\subsection{Equivalent cameras method}

In order to determine ultrasonic responses of exoenzymatic processes in biological wastewater treatment and treatment of sediments, ultrasonic proximity sensors for determining resonance in an equivalent chamber are used.

The configuration of identification pacemakers of ring structures of the corresponding enzymatic process is recorded by high sensitivity microcams.

By the method of equivalent chambers (Figure 1), the cellular structure of the hexagonal geometric shape of equivalent chambers is used (1). Remote sensors are located on the faces respectively (2), which fix wave reviews in the covered equivalent volume. Depending on the degree of abundance of the cellular structure and the sensitivity of the remote sensor, the resulting complex of data on the enzymatic process is detectable. 


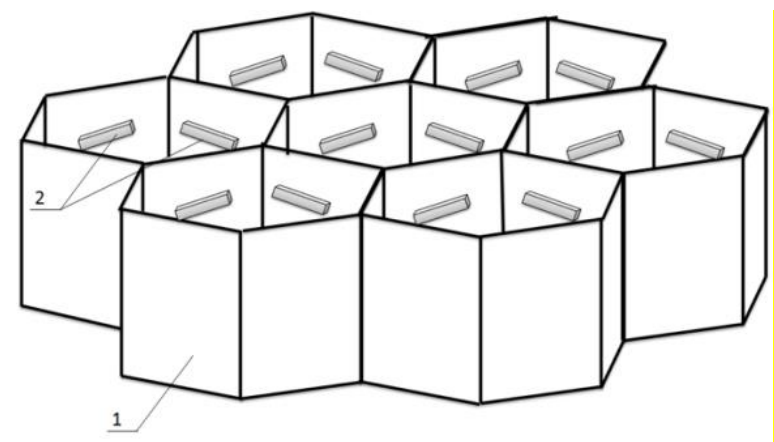

Fig. 1. Scheme of equivalent chambers for registration of wave responses of enzymatic processes: 1 equivalent chamber; 2 - remote sensing sensors for identifying wave characteristics.

The cell volumes are determined according to the identification wave characteristics of the enzyme process and depend on the sensitivity of the remote sensors. The processing of data from a complex of sensors (volumetric, induction, optical, ultrasonic, thermal) creates an integrated identification process characteristic and the morphology of the wave response.

Software development is formed on certain identification wave characteristics of enzymatic processes and features of resonances or absorption corresponding to this process.

\subsection{Probability of wave response}

Based on the laws of distribution of random variables, the intensity of wave resonances in biological systems, it is possible to conditionally be divided into three stages. The first stage is when there is biomass growth and the value of the observation coefficient $\left(\mathrm{Y}_{\mathrm{obsr}}\right)$ increases to 0.22 , respectively. Then, in the second stage, the intensity of the wave resonances has a constant oscillation (exponential law). The third stage: according to the normal law, in connection with the slowing down of metabolic processes, or "aging" of biomass, the decrease in the wave resonance of the growth observation coefficient is observed (Figure 2).

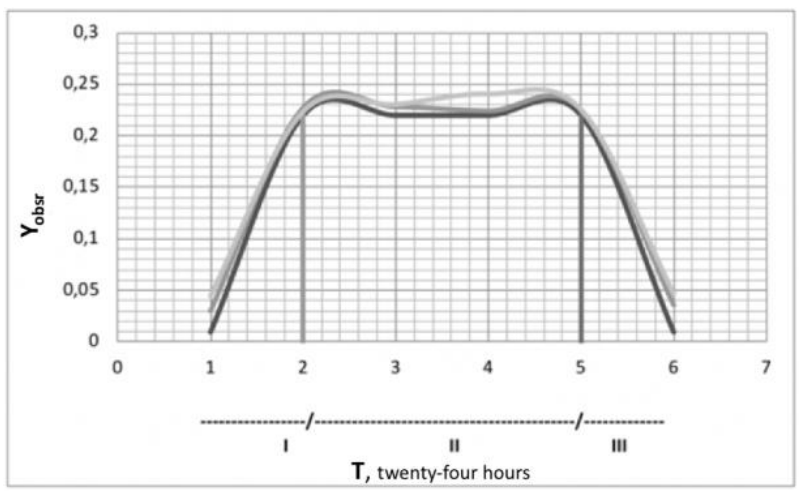

Fig. 2. Intensity of wave resonances of enzymatic processes in biological systems of water treatment reactors and treatment of precipitation: I - stage of biomass growth; II - constant oscillation stage; III - "aging" of biomass.

The probability of the wave response depends on the intensity of the wave resonance and has a corresponding maximum and minimum, respectively (Figure 3). 


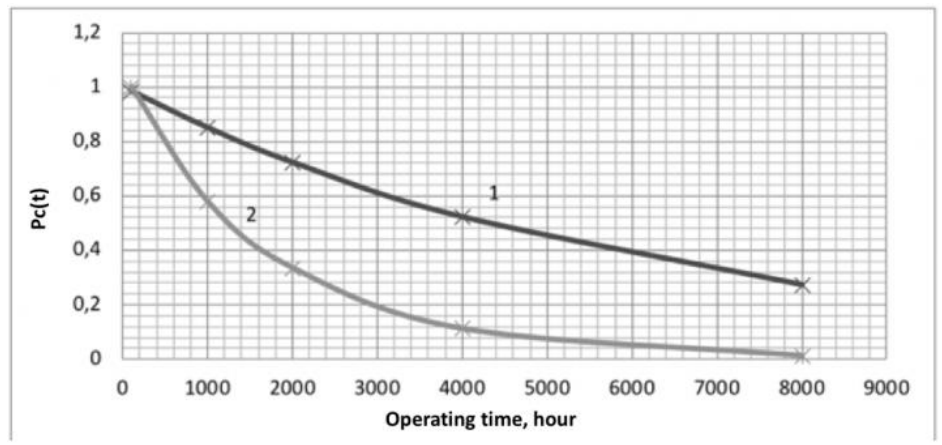

Fig. 3 Probability of the wave response of enzymatic processes in biological systems of water treatment reactors and treatment of precipitation: 1 - maximum; 2 - minimum.

The registration of the system's wave response will make it possible to optimize the operation of any biological wastewater treatment reactor from high molecular weight organic compounds and also to optimize the intensification parameters for the process by various types of wave action on the microbiological system in accordance with the wave resonance arising in response.

- According to selectivity to biochemical processes, the wave response of homogeneous processes of enzymatic hydrolysis of high-molecular organic compounds during biological water purification appears.

- The probability of a wave response corresponds to the laws of distribution of random variables in accordance with the three stages of operating time.

- Registration and identification of wave reviews of biochemical processes of biological water purification from high molecular organic compounds will provide an opportunity to optimize the operation of biological reactors of various types and intensification by various types of wave nature according to the value of the wave resonance.

- The identification of the wave response with respect to the selectivity of the enzymes released by the bacteria on the corresponding substrate makes it possible to determine the magnitude of the wave action to increase the enzyme concentration due to the wave resonance.

\section{Conclusion}

Based on theoretical studies, the hypothesis is advanced on the existence of a wave response of enzymatic reactions of aerobic and/or anaerobic conversion of organic substrate, hydrolysis of high molecular weight organic compounds, nitrification, denitrification, biological phosphorus removal in biological systems of water treatment and treatment of precipitation. It is proposed to use the methodology of resonant phenomena to determine the morphology of the wave characteristics and the equivalent chamber method for identifying wave responses of enzyme reactions in the reactor volume. On the basis of the laws of distribution of random variables, the probability of the wave response of the intensity of the appearance of wave resonance is determined for the mathematical description of the corresponding model systems.

The further research tasks are also determined:

- Obtaining the identification of wave responses of model biological systems of enzymatic reactions of aerobic and/or anaerobic conversion of organic substrate, hydrolysis of high 
molecular weight organic compounds, nitrification, denitrification, and biological phosphorus removal;

- Determining the morphology of wave characteristics by the equivalent volume method;

- Obtaining the parameters of resonance phenomena in the ultrasonic study of biological reactor systems.

The practical value of these studies is to unify the evaluation of the efficiency of water treatment reactors and treatment of precipitation in a stationary state and in an excited stateб as well as to determine the degree of exposure (hydrodynamic, thermal, magnetic, electromagnetic, low-frequency ultrasonic).

\section{References}

1. M. Heinze, Wastewater treatment (Mir, Moscow, 2008)

2. Yu. A. Stepkina, Building Engineering, 1, 44-49 (2007)

3. L. O.Nikiforova, L. M. Belopolsky, I. A. Pavlova, Use of biocoagulation at sewage treatment from connections of manganese (Chisinau, 2002)

4. U. Wiesmann, S. Choi, E.-M. Dombrowski, Fundamentals of biological wastewater treatment ( Wiley-VCH Verlag, KGaA, Weinheim, 2007)

5. A. B. Rubin, Biophisics (Universotet Book House, Moscow, 1999-2000)

6. I. M. Aksenova, Science-Technical Proceedings, 24 (2014)

7. I. M. Aksenova, Science-Technical Proceedings, 26 (2016)

8. E. S. Saverina, Biochemistry (GEOTAR-Media Publishing Group, Moscow, 2016)

9. V. D. Dmitriev, Operation of water supply systems for sewage and gas supply: a directory (Stroiizdat, Moscow, 1988)

10. E. A. Vorobyev, Theory of ultrasonic vibrations as a basis for constructing and applying technical means of obtaining information (SPbGUAP, St. Petersburg, 2002)

11. V. S. Kononenko, Acoustic Journal, 31, 6 (1985) 